\title{
Improved FNA cytology results with a near patient diagnosis service for non-breast lesions
}

Frederick Mayall, Aran Denford, Bridget Chang, Ann Darlington

\begin{abstract}
Aim-To review fine needle aspiration (FNA) cytology from sites other than the breast a year before and a year after the introduction of a near patient FNA diagnosis (NPFD) service in which the FNA were performed by a pathologist and reported within a few minutes.

Methods-The setting was a large hospital in rural New Zealand. The year before the introduction of the NPFD service was examined retrospectively, and the year after prospectively. The pattern of use and the quality of the results before and after starting the NPFD service were compared.

Results-Time taken to report the specimens decreased from a few days to a few minutes. There were statistically significant changes in the following: an increase from 237 to 304 in the number of nonbreast FNA performed, and in particular an increase from 65 to 113 in the number for general surgery; an increase in the use of immunolabelled flow cytometry from 0 to 19 and cell blocks from 3 to 41 ; an increase in specificity from $53 \%$ to $80 \%$; a decrease in the overall inadequacy rate from $29 \%$ to $9 \%$; and a decrease in the inadequacy rate for cancers from $9 \%$ to $2 \%$. The cost of the non-breast FNA service increased by about $£ 9200$ a year. Conclusions-Starting an NPFD service for sites other than the breast greatly reduced the reporting time and produced statistically significant increases in the use of FNA cytology and in the quality of the results.

(f Clin Pathol 1998;51:541-544)
\end{abstract}

Keywords: near patient diagnosis; fine needle aspiration cytology; audit

In this study we reviewed the pattern of use and quality of fine needle aspiration (FNA) diagnoses from sites other than the breast a year before and a year after the introduction of a near patient FNA diagnosis (NPFD) service. The setting was a large general and tertiary referral hospital (750 beds) servicing approximately 550000 people in a rural area of New Zealand.

\section{Methods}

The first year was examined retrospectively and the second year - after the introduction of the NPFD service-was examined prospectively. The information collected for each case included the case number, the patient identifi- cation, the clinical specialty, the FNA operator, the FNA reporter, the cytological diagnosis, the results of any histology related to the FNA sampled lesion, and the follow up from the clinical notes for those lesions with no clear final diagnosis. In our laboratory the cytological diagnosis is coded according to the NHS Breast screening programme guidelines for cytology procedures and reporting in breast cancer screening (available from NHSBSP publications, Fulwood House, Sheffield S10 3TH, UK): C1, inadequate; $\mathrm{C} 2$, benign; $\mathrm{C} 3$, atypical; C4, suspicious; C5, malignant. These codes and the related performance statistics are comprehensively defined and widely understood. They were originally intended to apply to breast cytology but they are applicable to all FNA cytology. The significance of differences between the first and second years was tested with the $\chi^{2}$ test with Yates' correction using a computer program "Chi-square V2.0" from Southampton University Department of Medical Statistics and Computing. For those tests in which the values were small the computer program automatically selected Fisher's exact test. Some of the figures needed to be compared using a test of difference in the frequency of an event in a large population assuming a Poisson distribution (table 1). Probability (p) values less than 0.05 were accepted as significant.

In the year before the introduction of the NPFD service the FNA were performed by clinicians and their technique varied. Most used a needle gun device to apply suction. Usually the needles were between 18 and 23 gauge. Most clinicians sent an air dried slide for a

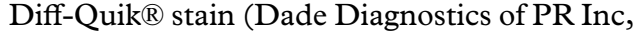
Aquada, Puerto Rico, USA) and a cytofixed slide for Papanicolaou stain. These were reported in the next few days by any one of six histopathologists rotating on a roster. They had varied levels of experience and interest in cytopathology. After the introduction of the NPFD service the FNA were performed by one of two mobile pathologists with an interest in FNA cytology, except for image guided FNA, when the needle was handed to one of these pathologists for preparation of the slides after the radiologist had aspirated the lesion. The FNA performed by the cytologist were done with a needle-only technique using a 23 or 25 gauge 1.25 inch $(3 \mathrm{~cm})$ needle. The slides were air dried, often aided by a small hair dryer, and Diff-Quik stained (fig 1). The slides were then examined with a portable Olympus CHK microscope (weight $4100 \mathrm{~g}$ ) and a hand written report issued within a few minutes. The slides were taken back to the laboratory to be mounted, examined again, and a final compu- 


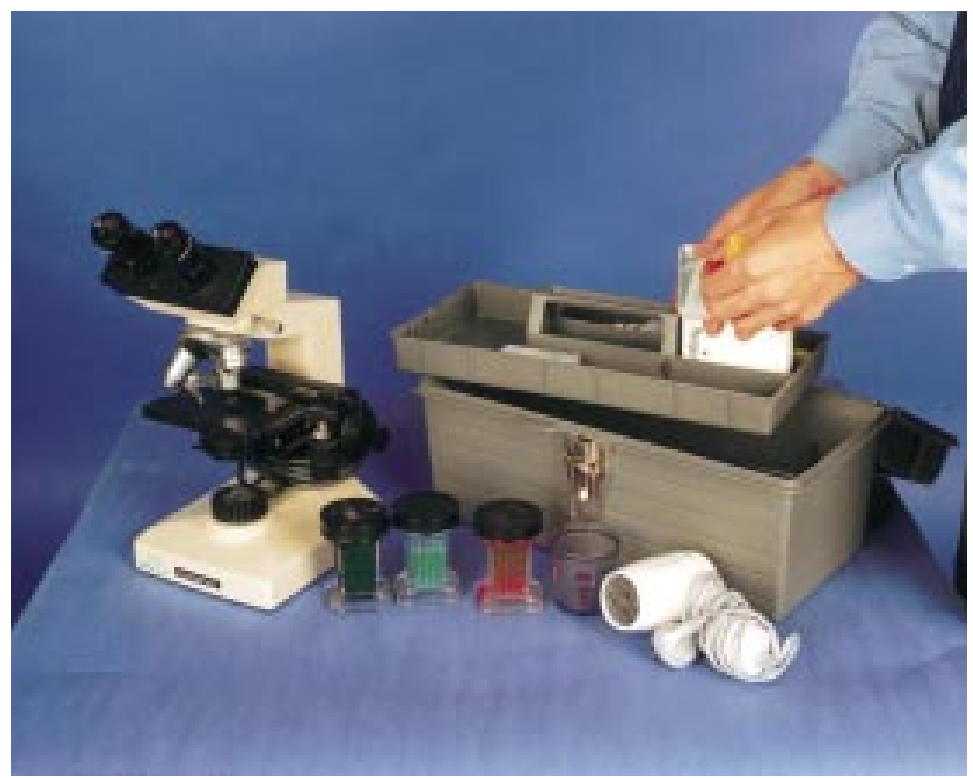

Figure 1 A light weight microscope and a fishing tackle box containing the aspiration and staining equipment including a small hair dryer.

ter printed report issued. The final report " $C$ " code was used in this study. This was rarely different from the initial "C" code.

The introduction of the NPFD made it possible for additional material to be gathered for special investigations, in particular cell blocks for immunohistochemistry and immunolabelled flow cytometry for the diagnosis and typing of lymphomas. These samples were taken by a second FNA of the lesion within a few minutes of the first specimen having been examined. The flow cytometry results were generally available on the same day, while cell block immunostains took one or two days.

The cost of the service was calculated from a schedule of prices that the private laboratories in New Zealand are paid by the Regional Health Authorities for items of service. A routine FNA is priced at NZ\$34 and an FNA taken by the pathologist is NZ\$104. The actual cost is probably less.

\section{Results}

The time taken to report the cytology specimens decreased from a few days to a few minutes with the introduction of the NPFD service. The data concerning the pattern of usage and quality of results are given in table 1 . The " $\mathrm{C} 1$; inadequate" diagnoses were all a consequence of poor cellularity rather than poor preparation or staining. Almost all of the NHSBSP performance statistics show a trend towards improved results with the introduction of the NPFD service, and some of these were statistically significant. The C code of the initial diagnosis was very rarely changed in the final diagnosis except in those cases in which immunostains and flow cytometry allowed a more detailed final diagnosis to be made. For example, B cell lymphomas were usually coded "C4; suspicious" initially and then " $\mathrm{C} 5$; malignant" when light chain restriction was found on immunolabelled flow cytometry. A "C2; benign" initial cytological diagnosis was never
Table 1 Fine needle aspiration (FNA) cytology of non-breast lesions a year before and a year after the introduction of a near patient diagnosis service

\begin{tabular}{|c|c|c|c|}
\hline & Year before & Year after & $p$ Value \\
\hline \multicolumn{4}{|l|}{ Totals } \\
\hline Non-breast FNA & 237 & 304 & $<0.005^{\star}$ \\
\hline Breast FNA & 405 & 709 & $<0.0005^{\star}$ \\
\hline Total FNA & 722 & 1013 & $<0.0005^{\star}$ \\
\hline \multicolumn{4}{|l|}{ Cytology code } \\
\hline $\mathrm{C} 1$; inadequate & 68 & 26 & $<0.0005$ \\
\hline $\mathrm{C} 2$; benign & 78 & 142 & $<0.005$ \\
\hline C3; atypical & 3 & 10 & NS \\
\hline C4; suspicious & 12 & 12 & NS \\
\hline C5; malignant & 76 & 114 & NS \\
\hline \multicolumn{4}{|l|}{ Use by specialty } \\
\hline General surgery & 65 & 113 & $<0.0005^{\star}$ \\
\hline Medicine & 54 & 54 & $N S^{\star}$ \\
\hline Oncology & 34 & 58 & $\mathrm{NS}^{\star}$ \\
\hline Plastic surgery & 8 & 17 & $\mathrm{NS}^{\star}$ \\
\hline Ear, nose and throat & 56 & 39 & $\mathrm{NS}^{\star}$ \\
\hline Others & 20 & 23 & $\mathrm{NS}^{\star}$ \\
\hline \multicolumn{4}{|l|}{ Use by site s $^{\star}$} \\
\hline Thyroid & 39 & 47 & $\mathrm{NS}^{\star}$ \\
\hline Salivary gland & 36 & 36 & $\mathrm{NS}^{\star}$ \\
\hline Lung & 27 & 32 & $\mathrm{NS}^{\star}$ \\
\hline Liver & 5 & 13 & $\mathrm{NS}^{\star}$ \\
\hline Others & 130 & 176 & $\mathrm{NS}^{\star}$ \\
\hline \multicolumn{4}{|l|}{ Image guided FNA $A^{\star}$} \\
\hline Total & 46 & 56 & $\mathrm{NS}^{\star}$ \\
\hline $\mathrm{C} 1$; inadequate & 6 & 5 & NS \\
\hline \multicolumn{4}{|l|}{ Special investigations ${ }^{\star}$} \\
\hline \multicolumn{4}{|l|}{ Immunolabelled flow } \\
\hline cytometry & 0 & 19 & $<0.0005^{\star}$ \\
\hline Cell blocks & 3 & 41 & $<0.0005^{\star}$ \\
\hline \multicolumn{4}{|c|}{ NHSBSP standised performance statistics for cytology } \\
\hline Absolute sensitivity & $86 \%$ & $90 \%$ & NS \\
\hline Complete sensitivity & $91 \%$ & $96 \%$ & NS \\
\hline \multicolumn{4}{|l|}{ Specificity (biopsy } \\
\hline cases only) & $55 \%$ & $85 \%$ & NS \\
\hline Specificity (full) & $53 \%$ & $80 \%$ & $<0.05$ \\
\hline \multicolumn{2}{|l|}{ Positive predictive } & $100 \%$ & NS \\
\hline \multicolumn{3}{|l|}{ Positive predictive } & NS \\
\hline \multicolumn{4}{|l|}{ Positive predictive } \\
\hline $\begin{array}{l}\text { value of } \mathrm{C} 3 \\
\text { False negative rate }\end{array}$ & $\begin{array}{l}0 \% \\
0 \%\end{array}$ & $\begin{array}{l}0 \% \\
2 \%\end{array}$ & $\begin{array}{l}\text { N/A } \\
\text { NS }\end{array}$ \\
\hline False positive rate & $4 \%$ & $0 \%$ & NS \\
\hline Inadequate rate & $29 \%$ & $9 \%$ & $<0.0005$ \\
\hline \multicolumn{4}{|l|}{ Inadequate rate for } \\
\hline Suspicious rate & $6 \%$ & $7 \%$ & NS \\
\hline \multicolumn{4}{|l|}{ Cost of non-breast FNA } \\
\hline FNA unit cost & $\mathrm{NZ} \$ 34$ & $\mathrm{NZ} \$ 104$ & N/A \\
\hline Total cost & $\mathrm{NZ} \$ 8058$ & $\mathrm{NZ} \$ 31616$ & N/A \\
\hline $\begin{array}{l}\text { Full time pathologist } \\
\text { equivalent }\end{array}$ & 0.08 & 0.32 & N/A \\
\hline
\end{tabular}

NA, not applicable; NHSBSP, NHS breast screening programme.

For values marked $\star$, the year before and the year after were compared by a test of difference in the frequency of an event assuming a Poisson distribution in a large population. All other values were compared for proportional difference by a $\chi^{2}$ test as described in Methods.

revised to a "C5; malignant" in the final report or vice versa.

\section{Discussion}

There was a significant increase in the total non-breast FNA and the examination of usage by specialty shows a significant increase by general surgery in particular. During the time of the study there was a slight increase in the activity of the outpatient departments and this may have made a small contribution.

There was a significant improvement in specificity (full). Specificity is a measure of the proportion of truly benign lesions that are 
diagnosed as "benign; C2" on FNA. The improvement probably resulted from the significant reduction in the inadequacy rate. The false "malignant; C5" cases were reduced from three in the first year to none in the second but this did not reach significance. These false positives were a case of organising pneumonia, a neurofibroma, and an adenoid cystic carcinoma "recurrence" that was in fact just reparative. There was an increase in false "benign; C2" cases from none to two, but this did not reach significance. The false benign tumours were a case of necrotic squamous carcinoma in the lung and a necrotic deposit of metastatic squamous carcinoma in the psoas muscle.

The significant increase in the use of FNA for breast and non-breast lesions following the introduction of the NPFD service is an indication of its popularity. There was a significant reduction in the proportion of " $\mathrm{C} 1$; inadequate" FNA and an increase in the proportion of "C2; benign" FNA. These two findings are probably interrelated as benign lesions are more likely to yield an inadequate sample than malignant ones. The reduction in the inadequacy rate probably reflected the technique of the pathologists as aspirators and also the fact that they could repeat the aspiration immediately if they were not satisfied with it.

The NPFD service was valued by the clinical staff mainly because a near patient diagnosis allowed them to deal with the patient immediately and at a lower cost than if the patient needed to be seen again to be given their results or needed a surgical biopsy. The clinicians could trust the initial diagnosis because it was rarely revised in the final report and no " $\mathrm{C} 2$, benign" diagnoses were revised to " $\mathrm{C} 5$; malignant" or vice versa in the final report. There were two false "C2; benign" (final) reports using the NPFD service but no false "C5; malignant" reports. The sparse distribution of the population in New Zealand was a special consideration in our study. For some patients a visit to the hospital requires a five hour drive and a night away from home. This increases the demand for a rapid result.

The importance of the FNA operator is well established in breast cytology. ${ }^{1}$ Cytologists reporting breast specimens are well aware that consultants and senior registrars perform much better than inexperienced junior staff, particularly in achieving an adequate specimen. Pathologists have been shown to be highly effective aspirators of breasts and non-breast lesions. ${ }^{2-4}$ We used a "needle only" FNA technique. This is some times known as fine needle sampling (FNS) rather than FNA. It has been shown by Rajasekhar et al to be as effective as conventional FNA. ${ }^{5}$ It does not seem to yield fewer adequate specimens although the volume of material obtained is apparently less. Rajasekhar's study showed an inadequacy rate of $21 \%$ for FNS and $22 \%$ for FNA from a case mix consisting mainly of lymph nodes, thyroids, and soft tissue lesions.

There have been other studies looking at the impact of pathologist aspirators and the impact of a NPFD service. These have been reviewed and discussed by Stanley. ${ }^{6}$ Saleh and Khatib found a decrease in the inadequacy rate from $46 \%$ to $24 \%$ with pathologist aspirators of mainly non-breast lesions. ${ }^{7}$ They also believed that their service provided significant financial savings, citing the cost of a CT guided FNA as US $\$ 1400$ compared with a surgical biopsy at US $\$ 7720$. Franzen and Zajicek obtained an inadequacy rate of $11.9 \%$ in benign lesions and $3.3 \%$ in malignant lesions. ${ }^{8}$ Pak et al found improved near patient diagnosis results in image guided FNA of lung lesions. ${ }^{9}$ Hall et al reviewed 795 FNA of thyroid nodules and found an inadequacy rate of $6.4 \%$ for pathologists compared with $15 \%$ for medical centre clinicians and $32 \%$ for community physicians. ${ }^{10}$ Duguid et al achieved an inadequacy rate of $13 \%$ in 294 NPFD and an inadequacy rate of only $3.3 \%$ for lesions that were eventually diagnosed as cancers. ${ }^{11}$ Brown and colleagues found that a service similar to ours gave a complete sensitivity of $96 \%$ together with a low level of inadequacy (about $7 \%$ ). ${ }^{2}$ Brown and Coghill also found that in performing NPFD on 490 breast patients over 12 months there was a saving of $£ 135544$, the expenses of the service being $£ 27290 .{ }^{12}$ Kocjan found a decrease in the inadequacy rate from $46 \%$ to $9 \%$ when FNA were done by a cytopathologist on mainly breast and lymph node lesions. ${ }^{13}$ In relation to breast lesions this was believed to have saved over $£ 100000$ in a year (396 breast lumps) owing to a reduction in the need for surgical biopsies. With the introduction of our NPFD service our estimated costs increased from NZ $\$ 8058$ for 237 FNA to NZ $\$ 31616$ for 304 NPFD, an increase of NZ\$23 548, which is about $£ 9200$. Based on these costs the pathologists' commitment increased about 0.2 full time equivalents.

The NPFD service allowed the pathologist to perform a second aspirate on difficult cases for special investigations. We recently reviewed 50 consecutive cytology cell block specimens. Most of these were from near patient FNA. There was adequate cellularity in 48 of these cell blocks. ${ }^{14}$ We have also reviewed (unpublished) our rapid diagnosis FNA of lymphoid lesions and found that a second FNA obtained enough material for immunolabelled flow cytometry in 21 of 29 cases. Most of the inadequate samples were from reactive nodes, probably because they were usually smaller than nodes containing lymphoma. The flow cytometry results were usually available within four hours of the aspirate being taken.

Our study shows that a NPFD service in a large general hospital improves the quality of cytological diagnoses, and previous studies suggest that the cost is a fraction of the savings from improved speed and accuracy. It leads to an increase in the use of FNA cytology by the clinicians who appreciate the speed with which a pathological diagnosis can be made. Our NPFD service became permanent after being trialed for the year of the study.

We would like to thank Dr Roger Marshall of the Department of Community Medicine, University of Auckland, NZ, for his help with the statistical tests. 
1 Barrows GH, Anderson TJ, Lamb JL, et al. Fine-needle aspiration of breast cancer: relationship of clinical factors to aspiration of breast cancer: relationship malignancies. Cancer cytology results

2 Brown LA, Coghill SB, Powis SAJ. Audit of diagnostic accuracy of FNA cytology specimens taken by the histopathologist in a symptomatic breast clinic. Cytopathology 1991;2:1-7.

3 Winship TD. Aspiration of breast cancers by the pathologist. Am f Clin Pathol 1969;52:438-40.

4 Schultenover SJ, Ramzy I, Page CP, et al. Needle aspiration biopsy: role and limitations in surgical decision making. $A m$ f Clin Pathol 1984;82:405-10.

5 Rajasekhar A, Sundaram C, Chowdhary T, et al. Diagnostic utility of fine needle sampling without aspiration; a prospective study. Diagn Cyopathol 1991;7:473-6.

6 Stanley MW. Who should perform fine needle aspiration biopsies? Diagn Cytopathol 1990;6:215-7.

7 Saleh HA, Khatib G. Positive economic and diagnostic accuracy impacts of on-site evaluation of fine needle accuracy impacts of on-site evaluation of fine needle aspiration
8 Franzen SL, Zajicek J. Aspiration biopsy in the diagnosis of palpable lesions of the breast. Critical review of 3479 consecutive biopsies. Acta Radiol 1968;7:241-62.

9 Pak HY, Yokota S, Teplitz RL, et al. Rapid staining techniques employed in fine needle aspirations of the lung. Acta Cytol 1981;25:178-84.

10 Hall TL, Layfield LJ, Philippe A, et al. Sources of error in fine needle aspiration of the thyroid. Cancer 1989;63:71825

11 Duguid HL, Wood RA, Irving AD, et al. Needle aspiration of the breast with immediate reporting of material. $B M \mathcal{F}$ 1979;ii: 185-7.

12 Brown LA, Coghill SB. Cost effectiveness of a fine needle aspiration clinic. Cytopathology 1992;3:275-80.

13 Kocjan G. Evaluation of the cost effectiveness of establishing a fine needle aspiration cytology clinic in a hospital out patient department. Cytopathology 1991;2:13-18.

14 Mayall F, Chang B, Darlington A. A review of 50 consecutive cell block preparations in a large general hospital. f Clin Path 1997;50:985-90. 